\title{
Giant Barkas Effect Observed for Light Ions Channeling in Si
}

\author{
G. de M. Azevedo, P. L. Grande, M. Behar, and J. F Dias \\ Instituto de Física da Universidade Federal do Rio Grande do Sul, Avenida Bento Gonçalves 9500, 91501-970, \\ Porto Alegre, RS, Brazil \\ G. Schiwietz \\ Hahn-Meitner-Institut, Abteilung SF4, Glienicker Strasse 100, 14109 Berlin, Germany
}

(Received 23 June 2000; revised manuscript received 12 December 2000)

\begin{abstract}
Measurements of the electronic energy loss are presented for ${ }^{4} \mathrm{He}$ and ${ }^{7} \mathrm{Li}$ ions channeling along the Si main axial directions at intermediate to high projectile energies. The Barkas effect, an energy-loss enhancement proportional to the third power of the projectile charge at high energies, is clearly separated from other processes. It reaches about $50 \%$ for $\mathrm{Li}$ ions channeling along the $\mathrm{Si}\langle 110\rangle$ direction. The observed Barkas contribution from the valence-electron gas is in fair agreement with the Lindhard model.
\end{abstract}

DOI: $10.1103 /$ PhysRevLett.86.1482

The energy loss of ions slowing down in matter has been investigated for many years because of its relevance for ion beam analysis, materials modification, and nuclear physics. Moreover, there exist fundamental issues concerning the underlying physical processes of the energy loss at low and intermediate projectile energies. At high velocities, the main energy-loss mechanisms (ionization and excitation) are qualitatively well understood. But important points related to energy loss in the polarization field are still unclear. For the weakly interacting fast light ions, the polarization field is given by the dielectric function of the medium. It corresponds to an enhanced electron density around positive ions and to a reduction around negatively charged particles. This effect yields a $Z^{3}$ contribution to the stopping power at high velocities ( $Z$ is the projectile charge), and thus, it depends on the sign of the projectile charge.

This so-called Barkas effect has first been observed by Barkas and collaborators [1] who found that the range of negative pions exceeds the one of positive pions with equal incident velocity. A direct determination of a relatively small Barkas effect has recently been performed using antiprotons and protons $[2,3]$. For heavier projectiles, other higher-order effects as well as shell corrections compete equally with the Barkas effect, and thus a clear and unequivocal separation of this effect from the others has not been achieved successfully yet [4]. Previously, channeling measurements of the Barkas effect [5,6] were performed under conditions where the so-called Bloch correction [7] is of comparable magnitude but of opposite sign as the Barkas contribution. The leading term of the Bloch correction is proportional to $Z^{4}$, and it is closely related to the restriction of reaction probabilities to a maximum of 1 per target electron. To overcome these problems, it is therefore essential to determine the magnitude of the Barkas effect in an alternative way, where sources of errors are minimized.

All theoretical models of the Barkas effect (reviewed by Basbas [8]) agree about the importance of the polarization of the medium in distant collisions, where ions act
PACS numbers: $34.50 . \mathrm{Bw}, 34.50 . \mathrm{Fa}, 61.85 .+\mathrm{p}, 79.20 . \mathrm{Rf}$

similar to photons. However, the importance of close collisions has been a subject of controversy in the literature [8]. Lindhard [9] stated that the close collisions may contribute nearly equally to the Barkas effect, because of the influence of the dynamical screening potential on electron-scattering cross sections. It is noted that most of the models (electron gas, harmonic oscillator, etc.) do not fully correspond to the physical situation found in the experiments. Further complications arise from the fact that most models need minimum cutoff impact parameters and that the Barkas effect is typically only a few percent of the total energy-loss cross section. This hampers any definite conclusions about the calculations.

Here we report on measurements of a strong Barkas effect under channeling conditions for $\mathrm{He}$ and $\mathrm{Li}$ ions impinging along the Si main axial directions. Such a channeling investigation has many advantages compared to previous measurements performed in amorphous targets or at a random direction. (a) The main contribution to the channeling stopping power stems from the valence electrons and exactly these electrons are sensitive to the polarization field. (b) Disturbing effects such as shell corrections (originating from finite target-electron velocities), electron capture, or other inner-shell effects that overshadow the Barkas term are strongly reduced. (c) Channeling conditions provide the best scenario for the applicability of electron-gas models. In this work, we apply improved experimental and theoretical methods in order to extract the Barkas contribution to the energy loss with high precision.

For the channeling measurements, we have used the Rutherford backscattering (RBS) channeling technique together with a SIMOX target (a Si crystal film on top of $\mathrm{SiO}_{2}$ ). The advantage of the present experimental arrangement is that it does not make use of thin self-supported films as the ones used in transmission measurements. In addition, the present technique allows for stopping power measurements down to low energies. Consequently, we were able to measure in a wide energy range between 100 
and $1500 \mathrm{keV} / u$. The SIMOX samples consist of $2000 \AA$ $\mathrm{Si}\langle 100\rangle$ crystal layers on top of $4000 \AA \mathrm{SiO}_{2}$ buried layers, produced in $\langle 100\rangle \mathrm{Si}$ wafers. For each experiment, the samples (produced at IBM, T.J. Watson Research Center, New York) were etched to remove the native oxide film on the surface using a 10\% HF acid just before the measurements. Immediately after the cleaning, they were mounted on a three-axis goniometer of $0.005^{\circ}$ precision. The energy-loss measurements were carried out at channeling as well as at random directions. The backscattered He or $\mathrm{Li}$ particles were detected by $\mathrm{Si}$ surface barrier detectors located at $170^{\circ}$ with respect to the incident beam. The overall resolution of the detection system was around $12 \mathrm{keV}$ for $\mathrm{He}$ and $20 \mathrm{keV}$ for $\mathrm{Li}$ ions. The energy difference between the $\mathrm{Si} / \mathrm{SiO}_{2}$ interface positions in the random and channeling RBS spectra gives the corresponding difference in the energy lost along the incoming path of the projectile (before the backscattering in a random direction). As described in detail in Ref. [10], this difference provides accurate mean energy-loss results for the selected channeling direction. It should be stressed that the mean energy loss was some tens of $\mathrm{keV}$ (much smaller than the projectile energy) and the energy-loss distribution did not show any significant departure from a Gaussian.

Under channeling conditions, the energy loss due to the $\mathrm{Si}$ inner-shell electrons is strongly suppressed, since the ion-flux distribution along a $\mathrm{Si}$ major axial direction has a peak in the middle of the channel (flux peaking). Hence, in contrast to a random direction, the mean energy lost by the projectile after passing a certain thickness $X$ is given by

$$
\Delta E=\frac{\int_{A} d^{2} \rho \int_{0}^{X} d x \frac{d E(\vec{\rho})}{d x} \Phi(\vec{\rho}, x)}{\int_{A} d^{2} \rho \int_{0}^{X} d x \Phi(\vec{\rho}, x)},
$$

where $A$ is the transversal area of the $\mathrm{Si}$ axial channel, $\vec{\rho}$ is the position relative to the center of the channel, and $\Phi(\vec{\rho}, x)$ is the ion-flux distribution at depth $x$ along the channeling direction. The flux distribution is obtained from the LAROSE Monte Carlo program [11] with thermal vibrations according to the Debye temperature of $490 \mathrm{~K}$. The energy loss per traversed distance $\frac{d E}{d x}(\vec{\rho})$ may be divided into contributions involving the $\mathrm{Si}$ electrons and the energy loss from projectile ionization and excitation. Both contributions can be obtained from the impact parameter dependent energy loss $Q$ in atomic collisions between the projectile in a charge-state $q$ with a neutral $\mathrm{Si}$ atom. Each of the contributions may be written as an incoherent sum

$$
\frac{d E}{d x}(\vec{\rho})=\sum_{q} f_{q} \sum_{i} \frac{Q^{(q)}\left(b_{i}\right)}{d},
$$

where $d$ is the interatomic distance along the axial channel and the charge-state fractions $f_{q}$ have been taken from experimental channeling results $[12,13]$. The perpendicu- lar projectile/atom distances $b_{i}$ depend on the geometry of each main axial channel in Si. The trajectory averaged electron density of atomic Si orbitals deviates by only $2.5 \%$ from its solid-state value. Solid-state effects due to the $\mathrm{Si}$ valence electrons, namely the partially compensating collective screening and plasmon excitation, have implicitly been accounted for by normalizing the mean energy transfers of all shells to yield the Bethe value as extracted from experimental solid-state data. Within first-order perturbation theory, the results of our method (without Bloch terms) agree with full band-structure calculations for protons in Si [14] to within about 3\% for the total stopping power as well as for the channeling energy loss.

The impact-parameter dependence of the energy loss in single collisions plays a very important role in the determination of the stopping power under channeling conditions. Here we adopt the unitary convolution approximation (UCA) as described in Ref. [15], which is an $a b$ initio calculation that includes the full Bloch correction. This UCA impact-parameter realization of the first-order and Bloch terms has been successfully checked against full first-order calculations [16] as well as highly accurate numerical solutions of the Schrödinger equation [15]. The parameters of the calculations are the same as the ones used in Ref. [17], where the model has been extended to account for bound projectile electrons by considering screened interaction potentials. It is noted that we have assumed that projectile electrons remain in the ground state when the target is excited and vice versa. Thus, we also neglect electron-electron interactions between bound projectile electrons and target electrons that result in enhanced ionization and excitation cross sections at high energies [18]. At these energies, however, the projectile electrons are nearly completely stripped off and, consequently, this antiscreening effect is of minor importance for the present energy-loss calculations.

In Figs. 1 and 2 the energy-loss results are displayed for channeling along the $\mathrm{Si}\langle 100\rangle,\langle 111\rangle$, and $\langle 110\rangle$ directions as a function of the ${ }^{7} \mathrm{Li}$ and ${ }^{4} \mathrm{He}$ projectile energies. The error bars represent statistical uncertainties. Part of the data for the $\langle 100\rangle$ direction were published previously $[19,20]$. The UCA results without (solid lines) and with the Barkas correction by Lindhard (dashed lines) are shown for comparison. This correction was implemented by using the average of the projectile charge state to the third power $\left\langle q^{3}\right\rangle$ and by considering the 4 valence electrons of $\mathrm{Si}$ (with $\omega_{p}=0.612$ a.u.) to be homogeneously distributed in the solid. The latter assumption means that the Barkas correction is the same for random and channeling directions. This assumption is well justified (see Ref. [21]) for the $\mathrm{Si}\langle 100\rangle$ and $\langle 111\rangle$ channels, because the electron density is nearly uniform across these channels. For the widest channel of $\mathrm{Si}$, namely $\langle 110\rangle$, the electron density is nonuniform. The maximum influence on the stopping power due to valence electrons weighted by the ion-flux distribution is about $25 \%$. Assuming this maximum influence, a lower 


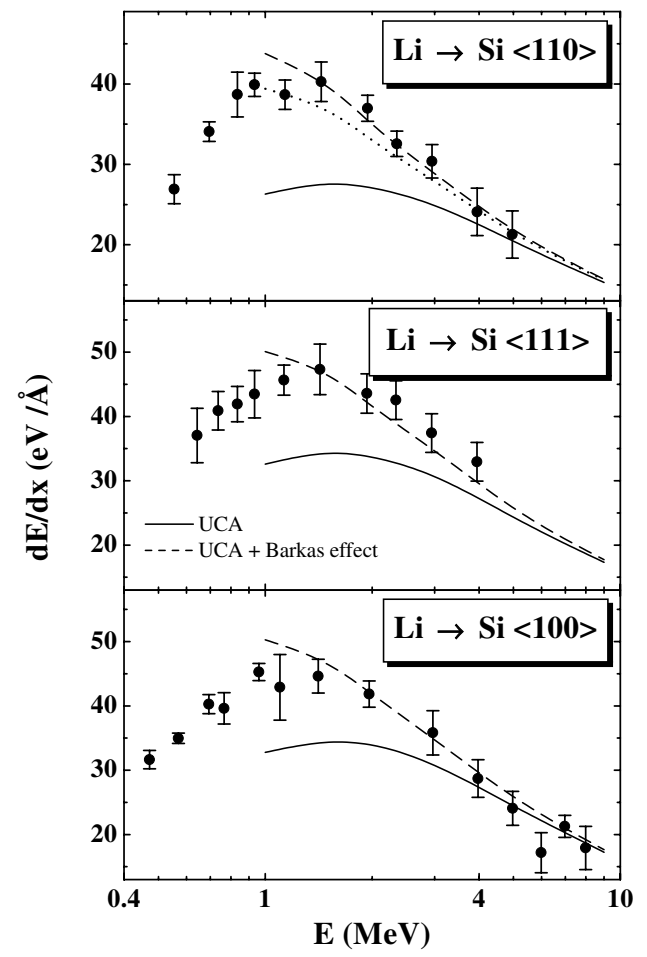

FIG. 1. Channeling stopping power as a function of the ${ }^{7} \mathrm{Li}$ energy for $\mathrm{Si}\langle 110\rangle,\langle 111\rangle$, and $\langle 100\rangle$ axial directions. The curves represent UCA calculations (solid lines) and the sum with the Barkas contribution according to Lindhard [9] (dashed lines). The dotted curve represents an estimate for the influence of the reduced valence-electron density in the $\langle 110\rangle$ channels.

boundary for the corresponding energy loss due to the reduced Barkas correction is shown by dotted lines.

The calculations have been performed only for energies down to about the stopping power maximum, because at low energies the influence of charge-exchange, multiple ionization, and shell corrections become important [21] even under channeling conditions. This would distort the interpretation of the Barkas effect. The lower limit for the use of the UCA calculations is about $150 \mathrm{keV} / u$, above which all-over uncertainties should be less than $15 \%$. As can be observed from Figs. 1 and 2, the Lindhard model accounts reasonably well for the polarization effects under axial channeling conditions. At energies below the stopping power maximum, a breakdown of the Lindhard model is expected, since it is based on perturbation theory.

It should be emphasized that the Barkas effect enhances the energy loss for $\mathrm{Li}$ around $1-2 \mathrm{MeV}$ by up to $48 \% \pm$ $15 \%$. This is by far the strongest Barkas "correction" that has ever been determined, and it would correspond to a ratio of 3 for the energy loss of $\mathrm{Li}$ and anti- $\mathrm{Li}$ with the same flux distribution.

The present experimental data as well as previous channeling data for $\mathrm{Li}$ and $\mathrm{He}$ ions (from Eisen et al. [22] and Lulli et al. [23] for $\mathrm{He}$ in $\mathrm{Si}\langle 111\rangle$ and $\langle 100\rangle$ directions and from Jiang et al. [13] for $\mathrm{Li}$ in $\mathrm{Si}\langle 100\rangle$ ) can all be presented in a single plot. This allows one to ver-

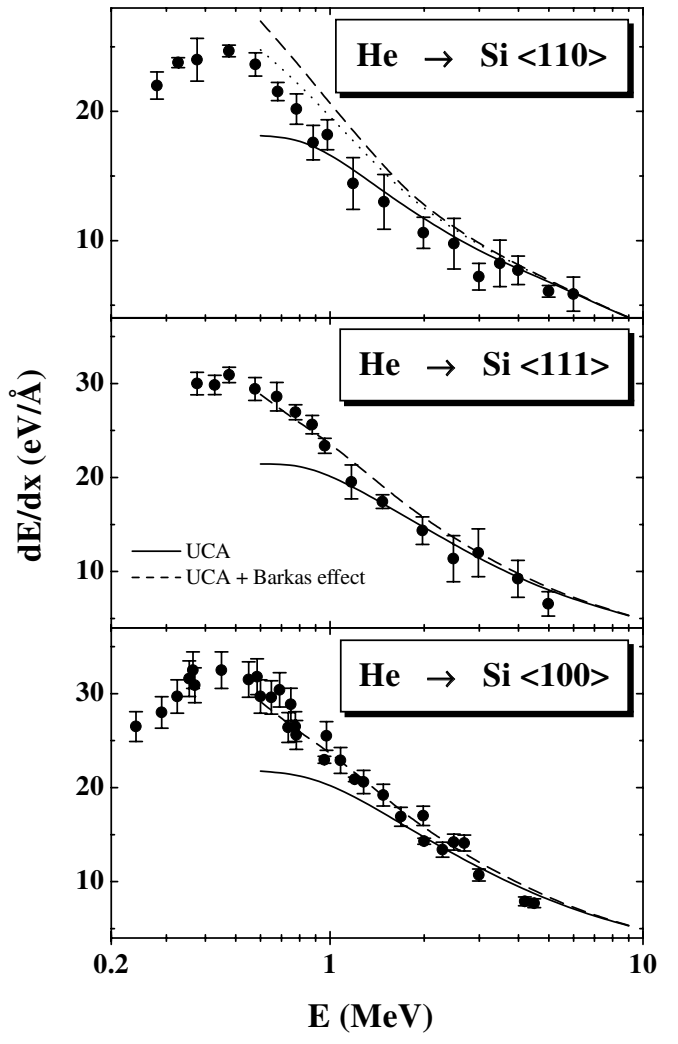

FIG. 2. The same as in Fig. 2, but for ${ }^{4} \mathrm{He}$ projectiles.

ify whether the observed differences from the UCA calculations (Bethe-Bloch) are due to the Barkas effect and not from other higher-order or shell corrections that do not scale with $\left\langle q^{3}\right\rangle$. Figure 3 presents all experimental channeling data after subtraction of the Bethe-Bloch term (through the UCA method) and division by the mean third power of the projectile charge state as a function of the projectile velocity. An inspection of this figure shows that the data are well grouped around an average curve (determined by a best fit), independent of the channeling direction and projectile specie. This gives a rather strong support to the interpretation of the Barkas term. The average curve agrees rather well with the Lindhard model. It should be stressed the previous channeling data have been measured using thick targets (about $1000 \mathrm{~nm}$ ). This fact may introduce a slight overestimation of the channeling energy loss due to dechanneling at crystal defects and electronic multiple scattering. The present experimental target thickness is small enough to prevent dechanneling effects but large enough to assure ion-flux equilibration.

It is pointed out that we also could have divided the data in Fig. 3 by the projectile nuclear charge state to the third power $\left(Z^{3}\right)$. Even in this case, we obtain the data well grouped around an average curve. However, the scaling factor $\left\langle q^{3}\right\rangle$ is more meaningful since it takes into account different projectile charges for distant collisions (outside the bound electron shell). The full inclusion of the projectile charge states for distant as well as for close collisions 


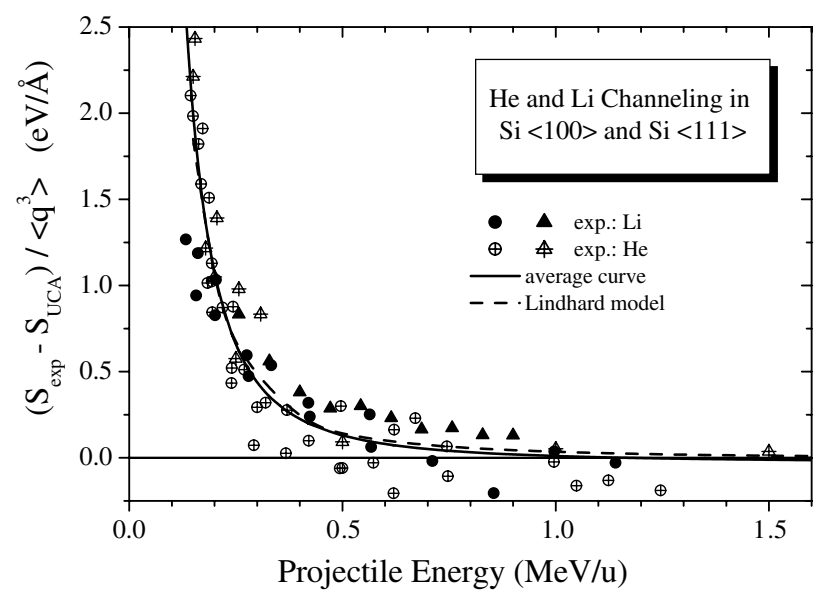

FIG. 3. Barkas contribution given by the difference of the experimental channeling data and UCA calculations for $\mathrm{Li}$ (closed circles) and He projectiles (open circles) in $\mathrm{Si}\langle 111\rangle$ and $\mathrm{Si}\langle 100\rangle$. Additional data from Refs. [13,22,23] are included as well (closed triangles for $\mathrm{Li}$ and open ones for $\mathrm{He}$ ions). The solid line is a fit to all experimental data, and the dashed line represents the Lindhard model.

is a very complex task. This calls for refined models that explicitly account for the screening due to valence electrons as well as bound projectile electrons.

Finally, an inspection of Figs. 1 and 2 shows that the Barkas effect is much smaller for He than for Li. This trend explains why the Barkas effect is so difficult to be observed with protons. For fast heavy projectiles with frozen charge states $(Z$ ranging from 9 to 17 at $3 \mathrm{MeV} / u$ ), Golovchenko et al. [6] have tried to determine the Barkas effect for best channeled ion trajectories along the $\mathrm{Si}\langle 110\rangle$ direction. However, they have obtained a strict $Z^{2}$ dependence of the stopping power, as a consequence of an almost complete cancellation of the $Z^{3}$ term with the $Z^{4}$ one at this velocity (proposed already in the original work [6]). This behavior was also verified by us using the UCA calculations and the Lindhard model for the Barkas contribution for the same best channeling conditions. Therefore, we take the present results as being the first true evidence and quantitative determination of the Barkas effect in a valence-electron gas.

In summary, we have determined the contribution of the Barkas effect for the Si valence electrons with channeled $\mathrm{He}$ and Li projectiles. The combination of the channeling RBS method and the recent theoretical realization of the impact-parameter dependence of the Bethe-Bloch contribution including screening has allowed for a clear quantitative determination of the Barkas effect. For Li projectiles and channeling along the $\mathrm{Si}\langle 110\rangle$ direction, the maximum polarization enhancement of the stopping power is about $50 \%$. At low incident energies, other effects such as electron capture, multiple ionization, and shell correc- tions come into play, and the present analysis method is not justified anymore. Within its range of validity, however, our analysis gives strong support to the Lindhard model.

This work was partially supported by the Brazilian agencies CNPq, CAPES, and by the program of BrazilianGerman cooperation PROBRAL 121/00.

[1] W. H. Barkas, N. J. Dyer, and H. H. Heckmann, Phys. Rev. Lett. 11, 26 (1963).

[2] L. H. Andersen, P. Hvelplund, H. Knudsen, S. P. Moller, J. O. P. Pedersen, E. Uggerhoj, K. Elsener, and E. Morenzoni, Phys. Rev. Lett. 62, 1731 (1989).

[3] S.P. Moller, E. Uggerhoj, H. Bluhme, H. Knudsen, U. Mikkelsen, K. Paludan, and E. Morenzoni, Phys. Rev. A 56, 2930 (1997).

[4] H. Bichsel, Phys. Rev. A 41, 3642 (1990).

[5] S. Datz, J. Gomez del Campo, P.F. Dittner, P. D. Miller, and J. A. Biggerstaff, Phys. Rev. Lett. 38, 1145 (1977).

[6] J. A. Golovchenko, A. N. Goland, J. S. Rosner, C. E. Thorn, H. E. Wegner, H. Knudsen, and C. D. Moak, Phys. Rev. B 23, 957 (1981); J. A. Golovchenko, D. E. Cox, and A. N. Goland, Phys. Rev. B 26, 2335 (1982).

[7] F. Bloch, Ann. Phys. (Leipzig) 16, 285 (1933).

[8] G. Basbas, Nucl. Instrum. Methods Phys. Res., Sect. B 4, 227 (1984).

[9] J. Lindhard, Nucl. Instrum. Methods 132, 1 (1976).

[10] J. H. R. dos Santos, P. L. Grande, M. Behar, H. Boudinov, and G. Schiwietz, Phys. Rev. B 55, 4332 (1997).

[11] J. H. Barrett, Phys. Rev. B 3, 1527 (1971).

[12] R. J. Petty and G. Dearnaley, Phys. Lett. 50A, 273 (1974).

[13] W. Jiang, R. Grötzschel, W. Pilz, B. Schmidt, and W. Möller, Phys. Rev. B 59, 226 (1999); 60, 714 (1999).

[14] J. M. Pitarke and I. Campillo, Nucl. Instrum. Methods Phys. Res., Sect. B 164-165, 147 (2000).

[15] G. Schiwietz and P. L. Grande, Nucl. Instrum. Methods Phys. Res., Sect. B 153, 1 (1999).

[16] P. L. Grande and G. Schiwietz, Phys. Rev. A 58, 3796 (1998).

[17] G. de M. Azevedo, P. L. Grande, and G. Schiwietz, Nucl. Instrum. Methods Phys. Res., Sect. B 164, 203 (2000).

[18] E. C. Montenegro, W.E. Meyerhof, and J.H. McGuire, Adv. At. Mol. Opt. Phys. 34, 249 (1994).

[19] J. F. Dias, G. de M. Azevedo, M. Behar, P. L. Grande, Chr. Klatt, and S. Kalbitzer, Nucl. Instrum. Methods Phys. Res., Sect. 148, 164 (1999).

[20] J. H. R. dos Santos, P. L. Grande, H. Boudinov, M. Behar, R. Stoll, Chr. Klatt, and S. Kalbitzer, Nucl Instrum. Methods Phys. Res., Sect. B 106, 51 (1994).

[21] P. L. Grande and G. Schiwietz, Nucl. Instrum. Methods Phys. Res., Sect. B 136-138, 125 (1998).

[22] F. H. Eisen, G. J. Clark, J. Bottiger, and J. M. Poate, Radiat. Eff. 13, 93 (1972).

[23] G. Lulli, E. Albertazzi, M. Bianconi, G. G. Bentini, R. Nipoti, and R. Lotti, Nucl. Instrum. Methods Phys. Res., Sect. B 170, 1-9 (2000). 